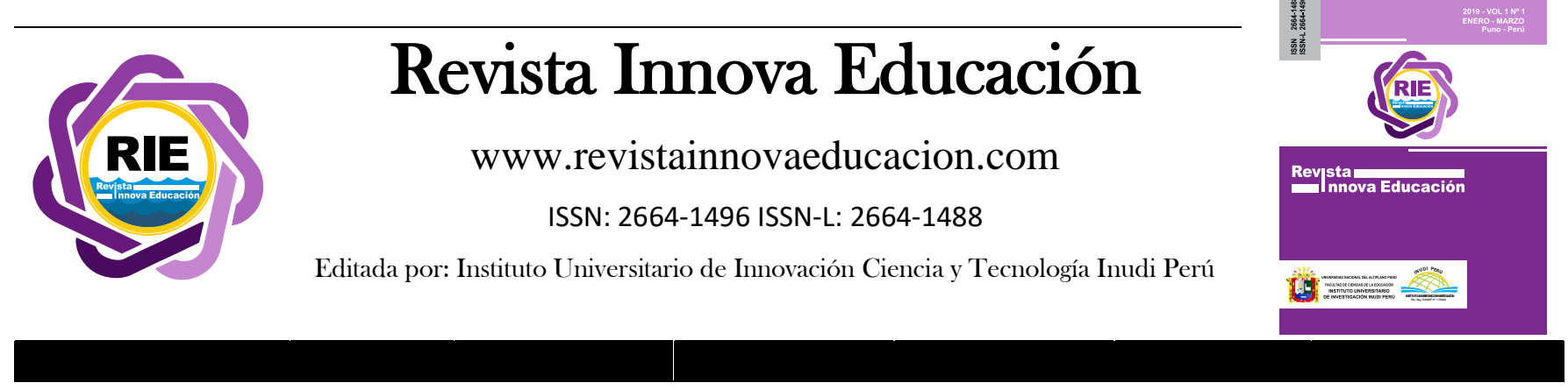

\title{
Valoración caósmica de la globalización en los sistemas educativos: una aproximación crítica desde la filosofía decolonial
}

\author{
Caósmica valuation of the globalization in the educational systems: a critical approach from \\ decolonial philosophy
}

\author{
Jesús Huanca-Arohuanca $^{1}$ (D) Wilson Sucari ${ }^{2}{ }^{\text {iD }}$; José Moriano ${ }^{3}$ iD ; Nancy Sapana-Valdivia $^{3}$ (D) \\ DOI: https://doi.org/10.35622/j.rie.2019.04.001
}

\footnotetext{
${ }^{1}$ Universidad Nacional de San Agustín de Arequipa, Perú

${ }^{2}$ Universidad Nacional del Altiplano, Perú

${ }^{3}$ Universidad Nacional San Antonio Abad del Cusco, Perú
}

\section{Recibido el 29/09/2019/ Aceptado el 18/10/2019/}

\begin{tabular}{l|l} 
ARTíCULO ORIGINAL & $\begin{array}{l}\text { El presente artículo reflexiona sobre las contradicciones de la valoración caósmica de } \\
\text { PALABRAS CLAVE }\end{array}$ \\
$\begin{array}{l}\text { la globalización en los sistemas educativos entonados y vistos desde la filosofía } \\
\text { decolonial. Siendo la investigación, de tipo documental bibliográfica y de examinación }\end{array}$ \\
teórico-conceptual, basado en la interpretación que da la orientación metodológica \\
globalización, \\
neoliberalismo, \\
política, educación
\end{tabular}$\quad \begin{aligned} & \text { la educación de los submundos emergentes basados en el planteamiento de la } \\
& \text { importancia de una enseñanza crítica humanizadora, centrada en la realidad de los } \\
& \text { estudiantes con cánones que respondan a la prognosis educativa, pero, que generen } \\
& \text { contradicciones racionales y eficientes frente a los que manejan la globalización. En } \\
& \text { conclusión, una educación basada en un modelo decolonial que no solamente está } \\
& \text { dedicado a instruir sino a enseñar el pensar crítico, resulta al final un peligro para el } \\
& \text { sistema-mundo capitaloceno, peligro que ha de nacer con ese pronunciamiento desde } \\
& \text { los que enseñan, reciben la enseñanza y desde los que aún no saben que se sumarán al } \\
& \text { proyecto del acto educacional con sentido censor. }\end{aligned}$

\section{KEYWORDS}

Philosophy, globalization, neoliberalism, politics, education

\begin{abstract}
This article reflects on the contradictions of the caósmica valoration of globalization in the educational systems intoned and seen from the decolonial philosophy. Being the research, of bibliographic documentary type and theoretical-conceptual review, based on the interpretation that gives the qualitative methodological orientation. In that vein, the results reveal the focus of the civilizing resistance on the education of emerging underworlds, based on the approach to the importance of critical humanizing teaching, focusing on the reality of students with canons responding to educational prognosis, but that generate rational and efficient contradictions against those that manage globalization. In conclusion, an education based on a decolonial model that is not only dedicated to instructing but teaching critical thinking, it turns out to be in the end a danger for the system - world capitaloceno, danger to be born with this pronouncement from those who teach, receive the teaching and from those who do not yet know that they will join the project of the educational act with a censor sense.
\end{abstract}

they will join the project of the educational act with a censor sense. 


\section{INTRODUCCIÓN}

Globalización es a buen seguro la palabra (a la vez eslogan y consigna) peor empleada, menos definida, probablemente la menos comprendida, la más nebulosa y políticamente la más eficaz de los últimos - y sin duda también de los próximos-años. Ulrich Beck (1998:40)

El neoliberalismo globalizante tradicional en términos doctrinales, era estudiado en tres dimensiones: como ideología, como forma de gobierno y como un conjunto de medidas económicas (Cárdenas, 2017). Sin embargo, en contextos actuales se pretende insertar una cuarta dimensión que es el sistema educativo. Ese sistema muy aclamado en coyunturas políticas pero que en la realidad es abandonado por los mismos. Evidentemente en esa línea, se puede catalogar al sistema educativo impartido en los submundos subyugados como una herramienta bifuncional: primero como herramienta de liberación, ya que éste se emplea bajo los estándares decoloniales, y segundo, como herramienta de dominio o control, porque, este tipo de educación está orientado a la expoliación/colonización del subconsciente. Bajo esa premisa, recordamos a Lyotard (1990) en la interpretación de Castro-Gómez (2007) cuando analiza que el primer meta-relato es el de la educación del pueblo, en esencia, todas las naciones tienen derecho a gozar de las ventajas de la ciencia y la tecnología, con el objetivo de «progresar» y mejorar las condiciones materiales de vida para todos. Asunto que, a los que mejor se acomodan a la globalización no les ha importado mucho. El segundo meta-relato para Lyotard es el del progreso moral de la humanidad. En este contexto, la función de la universidad o el sistema educativo ya no es tanto formar profesionistas, ingenieros, administradores o técnicos, sino formar humanistas, sujetos capaces de «educar» moralmente al resto de la sociedad. Asimismo, sujetos que puedan ser capaces de formar líderes espirituales dentro de sus naciones y que ayuden a visibilizar que el «otro» también existe-siente-piensa y que merece el respeto irrestricto.

La globalización es una forma de fundamentalismo instaurado a nivel planetario para someter al «otro», de ahí que, en los sistemas educativos el sujeto que habla siempre está escondido, se disfraza, se borra del análisis paraconceptual. A partir de ahí, la filosofía occidental ha privilegiado el mito del «Ego» no situado antes que el «otro», además, la ubicación epistémica étnica/racial/de género/sexual y el sujeto que habla están siempre desconectados. Conviene, sin embargo advertir que la filosofía y las ciencias occidentales pueden producir un mito sobre un conocimiento universal fidedigno que cubre y disfraza a quien habla así como su ubicación epistémica geopolítica y cuerpo-político en las estructuras del poder (Grosfoguel, 2006). Como es natural, el occidente domina y los subalternos plantean la antítesis con el diálogo intercultural entre el norte y el sur ontológico, en razón de romper los estereotipos de dominación. Sin duda, en este análisis uno de los problemas sigue siendo el paralelismo que alimenta la actitud impositiva para con el diálogo intercultural de saberes que busca la alternativa a la biocolonialidad del ser y desmerecer el hecho de estar impregnados en lógica del éxito individual (Beltrán, 2016). Razón por la cual, la globalización ha sido utilizado para justificar el progreso de la humanidad, 
volviéndose en ciertos niveles un dogma para aquellos que si habían sacado provecho del tema en cuestión.

A los ochenta se cataloga como la «década perdida» para América Latina (AL). Dicho periodo se adjudicó por los mayores ajustes estructurales/privatizadores de liberalización de mercados y desmantelamiento de políticas sociales. Así mismo, hay que mencionar, que casi todos los países de la región siguieron de alguna u otra forma las políticas del llamado «Consenso de Washington». De manera que, todos los sistemas educativos en su gran mayoría cegados por su escolástica y falta de criterio se colaron a tal imposición, como si de un acto divino se tratara. Pero, a finales de los noventa y la primera década del milenio, pareciera haberse reactivado el espacio del pensamiento crítico en la región y en las instancias educativas que en la actualidad se traduce como la filosofía decolonial. No obstante, lo que se ha escrito sobre estas dos décadas, estuvieron trabajados desde la perspectiva de la economía política marxista. Crítica que sigue siendo importante, pero insuficiente para dar explicación a nuevas tendencias tanto en la práctica social como en el campo de las ideas (Escobar, 2016). De ahí que, el objetivo del estudio es el de crear distancia respecto de la tradición eurocéntrica en los sistemas educativos, para abrir espacios analíticos en las realidades que han sido ignoradas o invisibilizadas, es decir, consideradas no existentes por la tradición crítica eurocéntrica que al final solo pueden ser recuperadas por la «sociología de las ausencias» (De Sousa, 2018) que se traducirá en otro concepto denominado «filosofía-educacional de las ausencias».

\section{MÉTODO}

El estudio se desenvuelve bajo una metodología cualitativa de tipo documental-bibliográfica y de revisión teórico-conceptual basado en la hermenéutica. De manera consecuente, el documento rastrea las diversas fuentes de mayor impacto en la base de datos como: Scopus, WOS, Thomson Reuters, SciELO, Eric, Redalyc, Dialnet, Latindex, Doaj y OEI. Revelando los resultados de esa manera, el foco de la resistencia civilizatoria en las esferas de la globalización caósmica se encuentra en ascenso. Planteamiento que, de ante mano, enfatiza la importancia de una enseñanza crítica humanizadora y que resalte la enseñanza liberadora conceptual-práctica. En efecto, también se presentan algunas propuestas teóricas recientes en torno al paradigma de investigación decolonial, las cuales rastrean los caminos posibles para la construcción de una postura desde y para la subalternidad que permita la incorporación metodológica, ontológica, ética y praxiológica de la ciencia y el conocimiento doxa en el campo académico (Canaza-Choque \& HuancaArohuanca, 2018; Rocha-Buelvas \& Ruíz-Lurduy, 2018; Huanca-Arohuanca \& Canaza-Choque, 2019).

\section{RESULTADOS Y DISCUSIÓN}

\subsection{Caósmos en los subalternos globalizados}

Si se analiza el fenómeno de la globalización desde la perspectiva filosófica, habría que constatar, en primer lugar, que lo global siempre ha sido un tema del que se ha ocupado la filosofía. En efecto, buscar los conceptos y principios universales que pueden considerarse válidos para la 
humanidad en su conjunto es parte de su tradición (Rohberck, 2018). En segundo lugar, la filosofía es la doctrina regulatoria de los marcos globalizadores que deterioran al sujeto ontológico y a la physis (naturaleza), dando a entrever que los siempre fulminados por el fenómeno en custodia son los que siempre carecen de hegemonía y dinero. Por ende, la globalización en los sectores emergentes se ha identificado como la negatividad sustancial del «otro» que provoca la desnaturalización y la dominación de X sobre Y. Sin embargo, vista desde la perspectiva de los que ostentan y saben controlar el fenómeno en cuestión, representa indudablemente desarrollo, libertad y democracia, conceptos muy bien manejados desde más de cuatrocientos años en que inició el sistema-mundo capitalista (Wallerstein, 2004), siendo además, el término más debatido e impreciso en los tiempos contractuales.

Como se puede ver, la evaluación caósmica está ubicado en dos vías. Por un lado, existen sociedades enteras que justifican y aceptan la globalización, sin darse cuenta de los efectos lamentables que puede provocar en determinados contextos. Por otro lado, hay otredades enteras que rechazan el fenómeno desgarrador, tales como los submundos supervivientes de AL, que han visto desde muy cerca la imposición y la masacre absoluta del colonizador disfrazado de globalizador. En esa línea, la educación se convierte en el eje vertebrador que presupone la crítica desde las aulas para con las aulas, para la búsqueda de no solo el diálogo, sino que también, la interpretación desde la perspectiva decolonial sin vórtice fundamentalista ni totalitarista. Entonces, hacia esa finalidad se pretende incidir hoy con la educación filosófica decolonial. Ya que de lo contrario ocurrirá lo de hace varios siglos, en el cual, dos mundos de la infancia y la adolescencia, se reconfiguraban en proposiciones como estas: «uno que se parece a la infancia idealizada de Rousseau que dedica su tiempo al juego y al estudio, y otro que se parece a la infancia de Dickens, la de niños y niñas que no pueden completar sus estudios» (Durán-Strauch, 2017, p. 889) por factores de alienación y de un simple determinismo económico, que al final se vuelve una simple aspiración envuelta a falacias propositivas de largo aliento.

El mundo de comienzos del siglo XXI necesita una decolonialidad que complemente la descolonización llevada a cabo en los siglos XIX y XX (Castro-Gómez \& Grosfoguel, 2007). Fundamento decolonial que se puede lograr con la ruptura epistemológica basado en la educación y la filosofía crítica ya muy bien trabajado por los teóricos de la filosofía de la liberación y el grupo decolonial. Entonces, para lograr este cometido se tiene que poner de manifiesto a la educación como única herramienta de combate ante la ciencia positivista que ha sepultado el conocimiento doxa. En esa medida, la filosofía y la educación son considerados como fenómenos taxativamente humanos, ya que, albergan varias potencialidades de orden crítico que no siempre han tenido ocasión de desplegarse a lo largo de la historia (Polo, 2018). Por ello, es preciso retomar el giro decolonial de Cesáire para romper el anhelo enfermizo que mantiene a los pueblos colonizados en la subordinación intelectual que fundamenta parte de la propia estructura colonial: la necesidad de definirse políticamente a través de los proyectos de liberación del blanco (Garcés, 2018) resumido como agente bélico globalizador.

Los humanos que habiten afro-indo-América deben entender de manera irrestricta que la globalización instaurado desde el norte para dominar el sur, es una mera y triste utopía de que se está rompiendo con las clases sociales o de que se está alcanzando el desarrollo anhelado para 
visibilizar a los que siempre habían estado en la oscuridad. Lo que se debe entender desde la escuela comúnmente confundido con Educación Básica, es que, desde el mismo lugar que supuestamente está proveyendo horizonte a la libertad, les están quitando la subjetividad de ser reconocidos en sus propios pensamientos y de alcanzar la genuina libertad. Entonces, cuando el afro-indo-americano pierde lo único que le quedaba de digno y ético, habrá sucumbido en la globalización constructo de aquellos agentes vampíricos de ese término. El humano consiente de la realidad ya descrita, debe buscar la elaboración de una teoría y práctica decolonial que pueda evidentemente asumir formas diversas, así como reconstruir y construir un pensamiento descolonizado, de la misma manera, resulta relevante en ese punto la necesidad de descolonizar otros ámbitos del contexto en el cual se desenvuelven aquellos pueblos, a saber, la historia, relaciones sociales y por cierto la política (Tricot, 2018).

El desarrollo de los pueblos tiene relación directa con el desarrollo de las personas que los conforman (Sanz \& Serrano, 2017). Pero, si eso no es así, entonces surge la idea de que el contexto habitado y estudiado no está siendo habitado por los que realmente deben habitar esos pueblos. Probablemente, ese habitad debería ser encontrado en la estabilidad necesaria de la educación bajo el sumak kawsay integrador de un discurso que va más allá del Buen Vivir, ya que al final, se vuelve una bandera de las resistencias y las movilizaciones indo-americanas. Entonces, parte de la lucha consiste en no dejarse asimilar a las concepciones occidentales ni convertirse en una versión étnica de la idea de bienestar utilizada por los organismos internacionales y el discurso publicitario. Esta cosmología suele ligarse más bien a posiciones decoloniales, reivindicativas de un estilo de vida autóctono (Papalini, 2017). En esa medida, surge la necesidad de la conformación del Nosotros, lo que sólo será posible en la medida en que abandonemos toda ilusión desarrollista, esto es, toda noción eurocéntrica y nos asumamos, más que decoloniales, anti-coloniales, más que antinorteamericanos, anti-imperialistas (Quintero, 2019), para optar como única vía, el diálogo entre culturas y la respuesta decolonial como epistemología del saber (Canales, Urrutia, \& Escudero, 2019).

Para su mejor tratamiento, se tiene que comprender en sentido estricto la babelización caósmica de la globalización, que produce relaciones de poder y de subjetivación paradójica para los sectores educativos emergentes. Ya que, está asociado a lo confuso y dicotómico. Ahora bien, la globalización, la filosofía y la educación son componentes muy mal explicados por los agentes educativos, debido a la falta de descolonización de la escuela. Además, desde el análisis preliminar de las fuentes primarias y secundarias a nivel latinoamericano, es posible identificar la mala aplicación de las políticas (Vera, Aguilera, \& Fernández, 2018) educativas. Se sabe desde la creación del Estado, que los sistemas imperiales imponen ciertos supuestos educativos como: la religión, la política educativa en diferentes niveles y la economía de mercado, para fulminar el subconsciente de los de abajo. Hoy al estudiante se capacita solo para trabajar mecánicamente y ya no se enseña a desarrollar ni entender que la educación es un espacio de formación de ciudadanos libres y conscientes, como diría Pablo Gentili. Para revertir el estado de la cuestión preliminar, la política y la educación deben ser los dos elementos cruciales en la conformación de las diferentes sociedades, pero estos elementos tienen que estar sometidos al debate (Medina, 2015). En ese sentido, la educación debe hacer que la política garantice y cumpla los requerimientos indispensables con los cuales debe disponer los agentes educativos en razones del 
cumplimiento del derecho a la calidad educativa. Pero, todo ello debe estar regulado por la filosofía que es la que da génesis y forma a las demás ciencias para su real y óptimo funcionamiento.

\subsection{Decolonizar la educación}

Decolonizar la educación desde el diálogo de saberes propuesto por el grupo decolonial resulta un aporte esencial para AL. Sin embargo, la construcción de la trasdisciplinariedad y transculturalización en los sistemas educativos sigue siendo imposible por los problemas que aquejan a los que brindan y reciben la educación con contenido europeísta/colonial, así mismo, el sujeto de la periferia conocido como «otro» u subalterno, no ha sido capaz de sacudirse del yugo colonizador y de la episteme occidental. De ahí surge la premisa de que «el subalterno no puede hablar» (Spivak, 2003, p. 362). En esa medida, el filósofo Santiago Castro-Gómez analiza el horizonte de las universidades y como afecta la hybris del punto cero a estos espacios educacionales, considerando

la idea de que la universidad pueda generar espacios en los que distintas formas de producir conocimiento - digamos entre la medicina indígena y la medicina tradicional— puedan coexistir, es, por ahora, una utopía, debido a que, de acuerdo con la taxonomía del punto cero, ambos tipos de saberes no son contemporáneos en el tiempo, aunque sean contemporáneos en el espacio. Recordemos que esa fue, precisamente, la estrategia colonial de Occidente, desde el siglo XVIII: el ordenamiento epistémico de las poblaciones en el tiempo. Unos pueblos, los más bárbaros, se hallan congelados en el pasado y no han salido todavía de su auto-culpable «minoría de edad», mientras que otros, los europeos civilizados y sus epígonos criollos en las colonias, pueden hacer uso autónomo de la razón y viven por ello en el presente. Aunque el médico indígena sea contemporáneo del cirujano que estudió en Harvard, aunque este último pueda saludarle y compartir con él un café, la hybris del punto cero lo clasificará como un habitante del pasado, como un personaje que reproduce un tipo de conocimiento «orgánico», «tradicional» y «pre-científico» (Castro-Gómez, 2007, p. 89).

En las esferas educativas aún se cree que la ciencia occidental es la única que debe enseñarse y plasmarse. Además, esta forma de pensamiento ha calado hasta tal punto en que los sujetos que lo estudian y reciben la enseñanza, admiten que lo cotidiano no tiene validez ni puede ser equiparable a la ciencia proveniente del occidente. Al mismo tiempo, se cree que la razón está muy por encima de la experiencia, a pesar de que estos dilemas ya han sido superados en la época moderna con Descartes (racionalismo) y Locke (empirismo). Por tanto, se puede precisar con cierta certeza que la hybris del punto cero no permite el diálogo de realidades y mucho menos la conexión entre educación práctica (fuera de las aulas) y la educación científica (dentro de las aulas). Es decir, la filosofía y la ciencia occidental se han negado a aceptar lo desarrollado en AL, silenciando e implantando de manera categórica diversas mallas curriculares para beneficio de los amos del mundo (capitalismo/neocapitalismo).

Adecuando el planteamiento de Castro-Gómez (2007) a la preocupación ya descrita a priori, se genera un vínculo semántico entre Universidad y el sistema educativo. Entonces, en vez de 
«decolonizar la universidad» se plantea ahora «decolonizar la educación» desarrollando y sintetizando dos planteamientos del autor en tratamiento:

1. El favorecimiento de la transdisciplinariedad. Decolonizar la universidad/educación significa, luchar contra la babelización y la departamentalización del conocimiento, firmes aliados de la lógica mercantil, a la cual se ha plegado la ciencia en el actual capitalismo cognitivo (p. 89).

2. El favorecimiento de la transculturalidad. La universidad/educación debería entablar diálogos y prácticas articulatorias con aquellos conocimientos que fueron excluidos del mapa moderno de las epistemes por habérseles considerado «míticos», «orgánicos», «supersticiosos» y «pre-racionales». Conocimientos que estaban ligados con aquellas poblaciones de Asia, África y AL, que entre los siglos XVI y XIX fueron sometidas al dominio colonial europeo (p. 90).

Reconceptualizando los dos criterios anteriores, se puede precisar que la globalización está de moda en todos los medios de comunicación, escritos-hablados, centros académicos, el mundo empresarial, los más altos niveles de los gobiernos y hasta en el seno de las familias más modestas. Esta súbita tendencia surge porque es la causa de los principales problemas, debido a la invasión de productos extranjeros, de menores costos y muchas veces supuestamente de mejor calidad que lo que se ofrecen en los pueblos originarios, ocasionando el cierre de empresas, desempleo masivo y angustia económica-social (Vivas, 1999). En esa medida, la educación también ha sufrido una serie de manipulaciones e imposiciones en todos los niveles. De ahí que, decolonizar la educación se vuelve una prioridad para los estratos sociales y culturales dominados de AL. Por ende, el primer planteamiento de Castro-Gómez está referido a la transdisciplinariedad de la ciencia en asuntos académicos que tiene una finalidad, que es, la desbabelización y desdepartamentalización de los sistemas educativos y al mismo tiempo ponerle frente al sistema capitalista/sexista/racista los conocimientos transversales de categoría doxa. El segundo argumento, está alusivo a la transculturalidad basado en el diálogo de saberes que debe superar la episteme del occidente. Frente a lo expuesto con anterioridad, tal vez suene como un alivio saber que América del Sur se despierta del yugo colonial, mientras, África se ve obligada a permanecer bajo la regla de oro del neo-colonialismo. Es más, mientras aumenta el número de líderes suramericanos que levantan su voz contra el imperialismo, ocurre todo al revés en el continente africano, tras la desaparición de los padres del panafricanismo y la liquidación de sus seguidores, lo cual incide en todos los órdenes (Nkogo, 2012).

Como se puede apreciar, la mundialización se ha impuesto en todos los sistemas económicos, ideológicos y educativos. Volviéndose a la vez, como una "Telaraña Mundial”, convertido para los Estados más poderosos un instrumento de vigilancia y de control global (Canaza, 2018b; Canaza-Choque, 2018) hacia quienes no logran entender el fenómeno expoliador. De ahí que lógicamente, se puede señalar que el primero, ha incrementado enormemente las ganancias y plusvalías, generando brechas sociales de todo tipo, mientras el segundo, ha servido a través de sus gobiernos como instrumento para alimentar al primero, pero el último es aún más preocupante, ya que, se ha hundido en lo amoral y poco a poco ha empujado a la gente a una etapa de deshumanización, generando nuevas disputas locales y regionales (Raventós \& Prats, 2012). Para 
los pueblos que no tienen las condiciones de insertarse a la globish (global) de la nueva comunicación planetaria, solo se esperan efectos negativos (Sorá, 2017). Así mismo, Para Dussel (2000: 52) a los 500 años del comienzo de la Europa moderna, en Informe sobre el desarrollo humano $1992^{1}$ de las Naciones Unidas encontramos que el $20 \%$ más rico de la humanidad (principalmente Europa occidental, Estados Unidos y Japón) consume el 82 \% de los bienes de la tierra, y el $60 \%$ más pobre (la «periferia» histórica del «Sistema-mundial») consume el 5,8\% de dichos bienes. ¡Una concentración jamás observada en la historia de la humanidad! ¡Una injusticia estructural nunca sospechada en la escala mundial! ¿No es este acaso el fruto de la Modernidad o del Sistema mundial que inició la Europa occidental?

En el transcurso de las dos últimas décadas, parte importante de los países Latinoamericanos han experimentado un giro político hacia la izquierda. Como ocurre en Venezuela, en Bolivia y en Ecuador, este giro ha significado redefinir el funcionamiento de las economías y de los sistemas políticos al interior de cada uno de esos países (Avendaño, 2010). Sin embargo, el sistema educativo no ha logrado el viraje hacia la decolonialidad como se planteaba y replanteaba por los teóricos de la subalternidad, a pesar de la existencia de una auto-descolonización, que actúa intentando globalizar la resistencia o darle por lo menos una movilidad continental a los pueblos naturales (Piñacué, 2014) como el caso aymarazo y Tía María en contra del capitalismo global en Perú (Canaza, 2018a). De esa manera, germinar la necesidad perentoria de propagar el pensamiento crítico conjuntamente con las preocupaciones centrales del desarrollo de las ciencias sociales latinoamericanas, resulta al final, una salida a las condiciones histórico-concretas que han vivido sus habitantes en el continente, los cuales expresan sus deseos de transformación, orientadas a ampliar la justicia y equidad social, la participación democrática para la conquista y ampliación de derechos para el afianzamiento de colectivos sociales emancipatorios (Asselborn, 2015).

\section{CONCLUSIONES}

La tarea de todos los intelectuales y agentes educacionales en el mundo fetichizado por la globalización, es sin duda, ayudar a comprender que el fenómeno en cuestionamiento es un instrumento de dominación que se sirve de la ciencia epistémica para lograr el mayor grado de control hegemónico, robando y colonizando la subjetividad genuina de los que habitan la periferia. Conforme a lo dicho, es que los humanos que habitan afro-indo-América deben buscar la descolonización en cada escala del subconsciente. Porque hasta este punto, la escuela ha perdido el horizonte contractual para lo que fue articulada, es más, las universidades como centros de conducción, también han extraviado la esencia del significado de su creación, y la lucha por la secularización de la escolástica enfermiza que dominó durante muchos siglos al parecer resulta insignificante. De modo que, se genera la certeza de decir que grandes centros pilotos como

\footnotetext{
${ }^{1}$ United Nations Development Programme (UNDP), Human Development Report 1992, Oxford University Press, Nueva York, 1992, p.35. En 1930 la diferencia entre los $20 \%$ más ricos de la humanidad y los $20 \%$ más pobres era de 1 a 30, en 1990 es de 1 a 59 (casi el doble en sólo 30 años). Véase también en el tema, The Invention of the Americas. Eclipse of "the Other" and the Myth of Modernity, Continuum Publishing, Nueva York, 1995; The Underside of Modernity. Ricoeur, Apel, Taylor and the Philosophy of Liberation, Humanities Press, Nueva York, 1996 y Etica de la Liberación en la Edad de la Globalización y la Exclusión, Editorial Trotta-UAM.I-UNAM, México, 1998.
} 
Universidades o colegios ya solo enseñan lo que al sistema o a los amos del planeta les interesa que aprendan.

La reflexión que presenta el tratado en sentido imperativo es, decolonizar la educación desde el diálogo de saberes en aras de la construcción trasdisciplinar y transcultural problematizado a partir del saber crítico, desde el cómo se piensa y qué se está pensando o se dice estar pensando de la realidad. El cual puede ser abordado desde la epistemología pluralista decolonial que, por un lado, hace referencia al reconocimiento de la heterogeneidad, multiplicidad y complejidad de epistemes que coexisten como un diálogo hermenéutico y por otro lado, una ruptura con el pensamiento europeo colonial superando la monocultura del saber científico por una interculturalidad emancipadora y una ecología de saberes, que emergen de la generación de conocimientos propios y autogestionarios (Iño, 2017). En concreto, decolonizar la educación significa rechazar la episteme y poner en trascendentalidad la doxa para escribir una nueva historia en cada esfera educativa y comprender tácitamente que la educación no es un sistema de capacitación para el trabajo, sino que es, la de formar ciudadanos libres y conscientes.

Recobrar la esencia de los espacios educacionales para que se convierta en el eje vertebrador de la sociedad moral, resulta construir el discernimiento sustancial crítico desde las aulas para con las aulas, en la búsqueda de no solo el diálogo, sino que también, la interpretación desde la perspectiva decolonial. He ahí, que entra la consonancia de los resultados, revelando el foco de la resistencia civilizatoria en la educación de los submundos emergentes, basados en el planteamiento de la importancia de una enseñanza crítica humanizadora, centrada en la realidad de los estudiantes con cánones que respondan a la prognosis educativa, pero, que generen contradicciones racionales y eficientes frente a los que manejan la globalización. En síntesis, una educación basada en un modelo decolonial que no solamente está dedicado a instruir sino a enseñar el pensar crítico, resulta al final un peligro para el sistema-mundo capitaloceno del que todos no quieren apartarse, peligro que ha de nacer con ese pronunciamiento revolucionario en sentido intelectual desde los que enseñan, reciben la enseñanza y desde los que aún no saben que se sumarán al proyecto del acto educacional con sentido censor.

\section{REFERENCIAS BIBLIOGRÁFICAS}

Asselborn, C. J. (2015). Fetishization and Critique of Reason. Literatura y Pensamiento Social, (2), 209-236. https://doi.org/10.1016/j.larev.2015.12.009

Avendaño, O. (2010). El buen vivir. Una vía para el desarrollo Alberto Acosta y Esperanza Martínez (comp.). Polis, Revista de La Universidad Bolivariana, 9(25), 557-561.

Beck, U. (1998). ¿Qué es la globalización? Falacias del globalismo, respuestas a la globalización. Barcelona: Paidós.

Beltrán, Y. J. (2016). Biocoloniality in exchanges between researchers of biodiversity and communities in Colombia. Tabula Rasa, (24), 213-240.

Canales, P., Urrutia, M. J., \& Escudero, F. (2019). Violence and Theory Pending Dialogues in the Colonial Context of the Mapuche People. Izquierdas, (46), 218-234.

Canaza-Choque, F. A. (2018). Educación y desigualdad en el Perú: rupturas y redes de esperanza en el plan de vivir juntos. UCV HACER Rev. Inv. Cult., 7(2), 69-79.

Canaza-Choque, F. A., \& Huanca-Arohuanca, J. W. (2018). Perú 2018: hacia una Educación 
$\begin{array}{lllll}\text { Intercultural } \quad \text { Bilingüe } & \text { sentipensante. } & \text { Sciendo, } & \text { 21(4), }\end{array}$ https://doi.org/10.17268/sciendo.2018.058

Canaza, F. A. (2018a). Justicia Ambiental vs Capitalismo Global Experiencias, Debates y Conflictos en el Perú. Rev. Investig. Altoandin 2018;, 20(3), 2018. https://doi.org/10.18271/ria.2018.368

Canaza, F. A. (2018b). La sociedad 2.0 y el espejismo de las redes sociales en la modernidad líquida. In Crescendo, 9(2), 221-247.

Cárdenas, J. (2017). Legal Theory and Neoliberal Globalization. Anuario de Filosofía y Teoría Del Derecho, (11), 215-272. https://doi.org/10.22201/iij.24487937e.2017.11.11075

Castro-Gómez, S. (2007). Decolonizar la Universidad. La hybris del punto cero y el diálogo de saberes. In El giro decolonial. Reflexiones para una diversidad epistémica más allá del capitalismo global. Bogotá: Siglo del Hombre Editores.

Castro-Gómez, S., \& Grosfoguel, R. (2007). El giro decolonial. Reflexiones para una diversidad epistémica más allá del capitalismo global. Bogotá: Siglo del Hombre Editores.

De Sousa, B. (2018). Introducción a las epistemologías del Sur. In Epistemologías del Sur. Buenos Aires: CLACSO.

Durán-Strauch, E. (2017). Derechos de niños y niñas: del discurso a la política local. Revista Latinoamericana de Ciencias Sociales, Niñez y Juventud, 15(2), 879-891. https://doi.org/10.11600/1692715x.1520623062016

Dussel, E. (2000). Europa, modernidad y eurocentrismo. In La colonialidad del saber: eurocentrismo y ciencias sociales. Perspectivas latinoamericanas. Buenos Aires: CLACSO.

Escobar, A. (2016). Sentipensar con la Tierra: Las Luchas Territoriales y la Dimensión Ontológica de las Epistemologías del Sur. 11-32. https://doi.org/10.11156/aibr.110102

Garcés, H. (2018). Racial Capitalism and Freedom Narratives: an approach to Cedric J. Robinson from Spanish state. Tabula Rasa, (28), 123-137. https://doi.org/10.25058/20112742.n28.6

Grosfoguel, R. (2006). Decolonizing Political-Economy and Post-Colonial Studies: Transmodernity, Border Thinking, and Global Coloniality. Tabula Rasa, (4), 17-48.

Huanca-Arohuanca, J. W., \& Canaza-Choque, F. A. (2019). Puno: Educación rural y pensamiento crítico. Hacia una educación inclusiva. Revista Helios, 3(1), 97-108. https://doi.org/10.22497/Helios.31.3106

Iño, W. G. (2017). Epistemología pluralista, investigación y descolonización. Aproximaciones al paradigma indígena. RevIISE, 9(9), 111-125. Retrieved from www.reviise.unsj.edu.ar

Lyotard, J.-F. (1990). La condición postmoderna Informe sobre el saber. México: Ediciones Rei.

Medina, J. (2015). Democratic education and educational policy: The education system discussed. Cuestiones Pedagógicas, 24, 103-116.

Nkogo, E. (2012). Aportes para una Filosofía Afro- Indo-Americana. FAIA, I, 1-8.

Papalini, V. (2017). The Well Living, between consumption tactics and ancient cosmogonies. Chasqui. Revista Latinoamericana de Comunicación, (134), 43-59.

Piñacué, J. C. (2014). Indigenous Thinking, Strains and Academy. Tabula Rasa, (20), 161-192.

Polo, J. (2018). La educación como herramienta de combate. De Sócrates a Paulo Freire. Areté Revista de Filosofía, 30(1), 163-188. https://doi.org/10.18800/arete.201801.008

Quintero, J. Á. (2019). La lucha por 'Ser Nosotros.' Utopía y Praxis Latinoamericana. Revista Internacional de Filosofía Iberoamericana y Teoría Social, 24(85), 282-293. https://doi.org/10.5281/zenodo.3338592 
Raventós, F., \& Prats, E. (2012). Knowledge Society and Globalisation. New Challenges for Comparative Education. Revista Española de Educación Comparada, 20, 19-40.

Rocha-Buelvas, A., \& Ruíz-Lurduy, R. (2018). Agendas for indigenous research and decoloniality. Izquierdas, (41), 184-197.

Rohberck, J. (2018). Globalization and History. Diánoia, 63(80), 119-147.

Sanz, R., \& Serrano, Á. (2017). Is education changing? A reflection on the function and meaning of school for everyone. Teor. Educ., 29(2), 167-184. https://doi.org/10.14201/teoredu292167184

Sorá, G. (2017). Traducción: potencial heurístico y desvíos teóricos de un tópico eficaz para pensar realmente la globalización. Revista de Estudios Sociales, 61, 38-39. https://doi.org/10.7440/res61.2017.08

Spivak, G. C. (2003). ¿Puede hablar el subalterno? Revista Colombiana de Antropología, 39, $297-$ 364. Retrieved from http://www.redalyc.org/articulo.oa?id=105018181010

Tricot, V. (2018). Mapuche Movement: Recovering Conventional Political Territory for the 21st Century. Izquierdas, (39), 252-272.

Vera, A., Aguilera, I. M., \& Fernández, R. (2018). Demands of authenticity: desire, ambivalence and racism in multicultural Chile. Convergencia Revista de Ciencias Sociales, (76), 13-36. https://doi.org/10.29101/crcs.v25i76.4500

Vivas, P. (1999). Globalización de la economía y/o mundialización del capital. Revista de La Facultad de Ciencias Económicas, 59-80.

Wallerstein, I. (2004). Retos para la universidad en el siglo XXI. Investigaciones Sociales, (13), 163-175. 